\title{
Sleep problems in autism may stem from leaky brain barrier
}

\author{
BY CHARLES Q. CHOI
}

23 JULY 2021

The sleep difficulties some autistic people experience may be due in part to leaks in the barrier that protects the brain from any toxins or pathogens in the blood, according to a new study in fruit flies.

The work also suggests that a behavioral treatment for chronic insomnia could help to alleviate sleep problems in some autistic people.

"Sleep restriction therapy is a first-line treatment for insomnia, but in the clinic, for genetic syndromes or neurodevelopmental disorders, it is hardly ever applied," says lead investigator Annette Schenck, professor of translational genomics of neurodevelopmental disorders at the Radboud University Medical Center in Nijmegen, the Netherlands. "We are hopeful that this can change in the near future."

Sleep problems occur in 50 to 80 percent of children with autism or other neurodevelopmental conditions, compared with roughly 20 percent of their neurotypical peers. To learn more about the roots of these problems, the researchers homed in on data from 27 people with mutations in CHD7 or CHD8, two closely related genes that are strongly linked to autism and sleep issues.

The data came from a sleep clinic, the Simons Simplex Collection, a repository of genetic and trait information from families with one autistic child, and a University of Washington study called The Investigation of Genetic Exome Research. (The Simons Simplex Collection is funded by the Simons Foundation, Spectrum's parent organization.)

Autistic people with mutations in CHD7 or CHD8 displayed significantly more difficulty falling asleep and staying asleep than roughly 2,300 autistic controls with no known autism-linked mutations, the study shows. And three people with CHD7 mutations and one person with a CHD8 mutation had chronic insomnia. 


\section{Glia surprise:}

The researchers next focused on Kismet, the version of CHD7 and CHD8 found in fruit flies. Insects with one mutated copy of the gene had reduced and fragmented sleep like that seen in people with $\mathrm{CHD} 7$ or CHD8 mutations. Suppressing Kismet expression only in neurons did not trigger any sleep issues, the researchers found, but quashing it in neuron-supporting glial cells during the flies' development did.

"Glia really get overlooked - they're the sad, neglected stepchildren of the brain," says Annika Barber, assistant professor of molecular biology and biochemistry at Rutgers University in New Jersey, who was not involved in the study. "This work shines a fascinating spotlight on a group [of cells] not often thought of in neurodevelopmental disorders."

The flies' sleep problems were specifically linked with subperineurial glia, a group of about 300 cells that help form the insect's blood-brain barrier.

"These cells have not previously been reported as a cause of sleep fragmentation," Schenck says. "Instead, it is well established that sleep loss impairs the function of blood-brain barrier in certain neurological disorders. So apparently, sleep and blood-brain barrier regulation are tightly intertwined, and so far we have known only half of the story."

Levels of the neurotransmitter dopamine, which is key to sleep and waking, were unaltered in the flies lacking Kismet in glia, further experiments showed. But those flies had unusually high levels of another neurotransmitter, serotonin, during development.

Using a drug to suppress the flies' serotonin levels during development eased their sleep difficulties. Increasing serotonin levels in wildtype flies during development led to sleep problems in adulthood. The results were published in June in Science Advances.

A condition called hyperserotonemia, characterized by elevated serotonin levels, is often linked with autism, although it remains unclear as to why. The new findings may renew interest in the role of serotonin in autism, especially in how it may breach the blood-brain barrier, says Georgianna Gould, associate professor of cellular and integrative physiology at the University of Texas Health Science Center at San Antonio, who did not take part in this work.

Mutations that knock out either CHD7 or CHD8 are extremely rare, but "they may be representative of more common blood-brain barrier deficiencies that stem from multiple other origins," Gould adds.

\section{Simple intervention:}

In a final round of experiments, the researchers developed an insect version of sleep restriction 


\section{Spectrum | Autism Research News}

https://www.spectrumnews.org

therapy, initially limiting the amount of darkness the insects experience and then gradually increasing it.

Controlling darkness and light in this way helped resolve the model flies' sleep problems, the team found.

Autistic people's sleep difficulties can exacerbate any cognitive problems, leading to more difficulties with sleep, says Krishna Melnattur, assistant professor of psychology and biology at Ashoka University in Sonipat, India, who was not involved in the work. "By showing that a simple behavioral modification can help reverse the sleep defects seen in Kismet mutant flies, this study offers a way to break this cycle."

Sleep restriction therapy is not standardized or uniformly applied for insomnia, making it difficult to gauge its therapeutic efficacy at this stage, Gould cautions. However, "there is ongoing research trying to identify the best approaches for this therapy," she says.

Future research should investigate how CHD8 mutations may affect melatonin, a molecule integral to sleep, Gould says. Previous work found melatonin supplements could help children with autism fall asleep faster and stay asleep longer.

In addition, scientists could explore whether Kismet mutations also lead to cognitive problems in fruit flies, and whether sleep restriction therapy might help alleviate some of these problems as well, Melnattur says.

"We also want to understand what CHD8, CHD7 and Kismet are doing in the glia and the bloodbrain barrier of humans and flies," Barber says. "From there, we can then explore potential pharmacological therapies, as opposed to just behavioral interventions."

Cite this article: https://doi.org/10.53053/UEHJ7006 